Internat. J. Math. \& Math. Sci.

Vol. 23, No. 2 (2000) 141-151

S0161171200000727

(C) Hindawi Publishing Corp.

\title{
ON BOUNDEDLY-CONVEX FUNCTIONS ON PSEUDO-TOPOLOGICAL VECTOR SPACES
}

\author{
VLADIMIR AVERBUCH
}

(Received 11 April 1997)

\begin{abstract}
Notions of a boundedly convex function and of a Lipschitz-continuous function are extended to the case of functions on pseudo-topological vector spaces. It is proved that for "good" pseudo-topologizers $\Psi$, any continuous $\Psi$-boundedly convex function is $\Psi$-differentiable and its derivative is $\Psi$-Lipschitz-continuous. As a corollary, it is shown that any boundedly convex function is Hyers-Lang differentiable.
\end{abstract}

Keywords and phrases. Filter, pseudo-topology, convergence space, pseudo-topologizer, pseudo-topological vector space, boundedly-convex, Lipschitz-continuous.

2000 Mathematics Subject Classification. Primary 26E15, 46A55.

1. Introduction. In [5] Joachim Focke, proved that for any continuous boundedlyconvex function $f$ on a Banach space $B$, its Fréchet derivative $f^{\prime}: B \rightarrow B^{*}$ (which always exists for such functions) is Lipschitz-continuous, that is, there exists $M>0$, such that for every $x_{1}, x_{2}$ from $B$,

$$
\left\|f^{\prime}\left(x_{1}\right)-f^{\prime}\left(x_{2}\right)\right\| \leq M\left\|x_{1}-x_{2}\right\| .
$$

Recall that a convex function $f: B \rightarrow \mathbb{R}$ is called boundedly-convex if there exists $M>0$ such that, for any $x_{1}, x_{2}$ from $B$ and any $\lambda_{1}, \lambda_{2} \geq 0$ with $\lambda_{1}+\lambda_{2}=1$,

$$
(0 \leq) \lambda_{1} f\left(x_{1}\right)+\lambda_{2} f\left(x_{2}\right)-f\left(\lambda_{1} x_{1}+\lambda_{2} X_{2}\right) \leq \frac{1}{2} M \lambda_{1} \lambda_{2}\left\|x_{1}-x_{2}\right\|^{2} .
$$

This means that the "deviation from linearity" for $f$ is not greater than for $(1 / 2) M\|\cdot\|^{2}$, in the case of a norm generated by a scalar product. See Remark 3.2. Notice that we can take $M$ from (1.2) as $M$ for (1.1).

We extend this result to the case of functions $f$ defined on arbitrary pseudo-topological (in particular topological) vector spaces (One often uses the term "convergence space" as a synonym to "pseudo-topological space". Below, we recall necessary definitions concerning pseudo-topological vector spaces. For more details, see [6].) For this end, we have to do three things:

(1) extend the notion of bounded convexity,

(2) extend the notion of Lipschitz-continuity,

(3) specify the definition of differentiability we use since, as it is well-known, the notion of Fréchet differentiability can be extended to the case of nonnormable spaces by many different ways.

We do these things by appealing the notion of a pseudo-topologizer, which was introduced in [4] and was thoroughly investigated in [1]. In those papers, it was attached 
to each pseudo-topologizer $\Psi$ a corresponding notion of differentiability (called $\Psi$ differentiability). Here, we attach to each pseudo-topologizer $\Psi$ notions of $\Psi$-bounded convexity and of $\Psi$-Lipschitz-continuity. Besides, we introduce an extension of the notion of bounded convexity (that does not append pseudo-topologizers). We prove that, roughly speaking, for "good" pseudo-topologizers $\Psi$, any continuous $\Psi$-boundedly convex function on a pseudo-topological vector space is $\Psi$-differentiable and that its derivative is $\Psi$-Lipschitz-continuous. Furthermore, we see that both the pseudotopologizer $F_{S}$ of convergence on a linear filter system $S$ and their onion modification $F_{s}^{\#}$ are "good". As a consequence, we derive that any continuous boundedly convex function on a topological vector space is Hyers-Lang differentiable.

The Focke's result corresponds to the case where $\Psi$ is the pseudo-topologizer $F_{b}$ of convergence on a system of bounded sets.

NotATION. Throughout, we use the following notation:

$\mathbb{R}$ the reals, $\mathbb{R}^{+}:=[0,+\infty)$

$I_{t}:=[-t, t], I_{t}^{+}:=[0, t]$ for $t \in \mathbb{R}^{+} ;$in particular $I_{1}=[-1,1], I_{1}^{+}=[0,1]$

$\mathbf{r}$ the filter of the neighborhoods of zero in $\mathbb{R}$

$\mathbf{r}^{+}$the trace of the filter $\mathbf{r}$ on $\mathbb{R}^{+}$

$X^{\#}$ the onion modification of $X$ (see below)

[A] the filter in a set $X$, generated by a subset $A \subset X$, i.e., the filter of all the subsets of $X$ that contain $A$

[x] the (trivial ultra-) filter generated by a singleton $\{x\}$

$F(X, Y)$ the set of all mappings from $X$ into $Y$

$L(X, Y)$ the set of all continuous linear mappings from $X$ into $Y$

If there enter filters in an expression, then this expression is to be interpreted as the image of the product of these filters by the corresponding mapping.

For example, if $x$ is a filter in a vector space $X$, then $\mathbf{r} x$ denotes the image of the filter $\mathbf{r} \times x$ (that is, of the filter with the basis consisting of all the products $I \times U$, where $I \in \mathbf{r}, U \in x)$ under the multiplication mapping $\mathbb{R} \times X \rightarrow X,(t, x) \mapsto t x$, that is the filter with basis consisting of sets of the form $I_{t} U$, where $t>0, U \in x\left(I_{t} U:=\left\{\tau x \mid \tau \in I_{t}\right.\right.$, $x \in U\})$.

As another example, if $\mathfrak{f}$ is a filter in $F(X, Y)$ and $x$ is a filter in $X$, then $\mathfrak{f}(x)$ denotes the image of the filter $x \times f$ under the evaluation mapping $X \times F(X, Y) \rightarrow Y,(x, f) \mapsto f(x)$, that is, the filter with basis consisting of all sets of the form $F(U), F \in \mathfrak{f}, U \in x(F(U):=$ $\{f(x) \mid f \in F, x \in U\})$.

Preliminary notions. A pseudo-topology (or a convergence structure) $\psi$ in a set $X$ is a mapping from $X$ into the power set of the set of all filters in $X$, that satisfies the following conditions (where we write $x \underset{x}{\downarrow} \psi$ instead of " $x \in \psi(x)$ "; one reads this relation as "the filter $x$ convergent to the point $x$ in the pseudo-topology $\psi$ "):

(a) $\forall x \in X:[x] \underset{x}{\downarrow} \psi$;

(b) $x_{1} \underset{x}{\downarrow} \psi, x_{2} \supset x_{1} \Rightarrow x_{2} \underset{x}{\downarrow} \psi$;

(c) $x_{1} \underset{x}{\downarrow} \psi, x_{2} \underset{x}{\downarrow} \psi \Rightarrow x_{1} \cap x_{2} \underset{x}{\downarrow} \psi$

A pseudo-topological space (or a convergence space) is the pair $(X, \psi)$, where $X$ is a set, and $\psi$ is a pseudo-topology in $X$. Usually, we simply write $X$ instead of $(X, \psi)$,

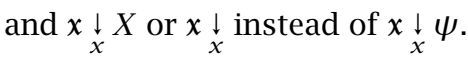


The pseudo-topology generated in a natural sense by a topology, is being identified with this topology.

A net $\left\{x_{\imath}\right\}_{\imath \in I}$ in a pseudo-topological space $X$ is said to converge to a point $x$ if $x \underset{x}{\downarrow} X$, where $x$ is the filter of "tails" of the net, that is $A \in x: \Leftrightarrow \exists \imath_{0} \in I: A \supset\left\{x_{\imath} \mid \imath \succ \imath_{0}\right\}$.

A mapping $f$ from a pseudo-topological space $X$ into a pseudo-topological space $Y$

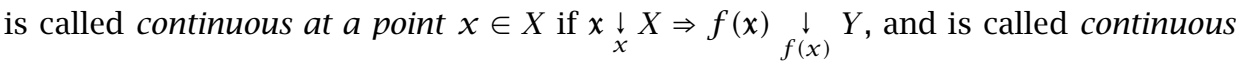
if it is continuous at each point. The pseudo-topology $\psi^{\prime}$ induced on a subset $X^{\prime} \subset X$ by a pseudo-topology $\psi$ in $X$ is defined as follows: $x_{x}^{\downarrow} \psi^{\prime}: \Leftrightarrow i(x) \underset{x}{\downarrow} \psi$, where $i$ is the inclusion mapping.

A pseudo-topological vector space (p.v.s.) is a vector space $X$ equipped with a pseudotopology that is compatible with the vector structure in $X$ (in the sense that the operations of addition and multiplication by a scalar are continuous, the real line being equipped with its natural topology).

If we weaken the compatibility conditions by replacing the requirement of continuity of the multiplication mapping by the following two conditions:

(a) $x \underset{x}{\downarrow} X, t \in \mathbb{R} \Rightarrow t x \underset{t x}{\downarrow} X$,

(b) $x \underset{0}{\downarrow} X, \mathfrak{t} \underset{t}{\downarrow} R \Rightarrow \mathfrak{t} x \underset{0}{\downarrow} X$,

we obtain the notion of a pseudo-topological vector group (p.v.g.). In p.v.s.'s it holds, besides, the condition

$$
\forall x \in X: \mathbf{r} x \underset{0}{\downarrow} X
$$

For p.v.g.'s, we simply write $x \downarrow$ instead of $x \underset{0}{\downarrow}$.

A filter $x$ in a p.v.g. is called bounded if $\mathbf{r} x \downarrow$. A set $B$ in a p.v.g. is called bounded if the filter $[B]$ is bounded (that is, if $\mathbf{r} B \downarrow$ ). A point $x$ in a p.v.g. is called bounded if the set $\{x\}$ is bounded (that is if $\mathbf{r} x \downarrow$ ). Thus, a p.v.s. is a p.v.g. such that all its points are bounded.

For every p.v.g. $X$, the associated onion (or equable) p.v.g. $X^{\#}$ is defined by the following conditions: as a vector space $X^{\#}$ coincides with $X$, and $x \downarrow X^{\#}: \Leftrightarrow(\exists y \downarrow X: x \supset$ $\mathcal{Y}=\mathbf{r} \mathfrak{Y})$.

It is clear that the pseudo-topology of $X^{\#}$ is stronger than the pseudo-topology of $X$, that is the identity mapping $X^{\#} \rightarrow X$ is continuous.

Any topological vector space (t.v.s.) $X$ is a p.v.s. and satisfies the condition $X^{\#}=X$.

\section{Pseudo-topologizers}

DEFINITION 2.1 [1]. Let $A$ and $B$ be subcategories of the category $P V G$ of all p.v.g.'s with the continuous linear mappings as morphisms. A pseudo-topologizer $\Psi$ on $A \times B$ is a covariant functor $\Psi: A^{\circ} \times B \rightarrow P V G$ (where $A^{\circ}$ denotes the dual category to $A$ ) that satisfies the following conditions:

(a) for any two objects $X$ and $Y$ from $A$ and $B$, respectively, $\Psi(X, Y)$ is (as a vector space) the vector subspace in $F(X, Y)$ that contains $L(X, Y)$;

(b) for any two morphisms $u \in L\left(X_{2}, X_{1}\right)$ and $v \in L\left(Y_{1}, Y_{2}\right)$ of the categories $A$ and $B$, respectively, and for any mapping $f \in \Psi\left(X_{1}, Y_{1}\right), \Psi(u, v)(f)=v \circ f \circ u$. 
For any pseudo-topologizer $\Psi$, the formula $\Psi^{\#}(X, Y)=\Psi(X, Y)^{\#}$ defines a pseudotopologizer $\Psi^{\#}$ which is called the onion modification of $\Psi$.

DefinITION 2.2 [1]. Let $A$ be a subcategory of $P V G$. We say that a linear filter system $S$ in $A$ is given if, for any p.v.g. $X$ from $A$, a nonempty set $S(X)$ of filters in $X$ is given such that the following conditions are fulfilled:

(a) for each $X$ and $Y$ from $A$, if $x \in S(X)$ and $l \in L(X, Y)$, then $l(x) \in S(Y)$;

(b) for each $X$ from $A$, if $x, y \in S(X)$, then $x+\chi \in S(X)$.

In the case where, for every $X$, all filters form $S(X)$ are filters of the from $[A]$, where $A$ is a subset of $X$, we say about a set system.

Important examples of linear filter systems are:

$B \cdots B(X)$ is the set of all bounded filters in $X$;

$C \cdots C(X)$ is the set of all convergent filters in $X$;

$b \cdots b(X)$ is the set of all bounded sets in $X$.

DeFINITION 2.3 [1]. Let $S$ be a filter system in A. We define the pseudo-topologizer $F_{S}$ (of convergence on $S$ ) on $A \times P V G$ by the conditions:

(a) $F_{S}(X, Y)=F(X, Y)$ and

(b) $\mathfrak{f} \downarrow F_{S}(X, Y) \Leftrightarrow \forall x \in S(X) \mathfrak{f}(x) \downarrow Y$.

LEMмA 2.4. Let $X$ be a p.v.g., let $Y$ be a p.v.s., and let $f: X \rightarrow Y$ be a continuous mapping. Then $f$ is a bounded point in $F_{c}^{\#}(X, Y)$, see [1].

Definition 2.5 (See [1]). We say that a pseudo-topologizer $\Psi$ on $A \times B$ possesses the property (EXP) if the well-known algebraical isomorphism

$$
F\left(X_{1} \times X_{2}, Y\right) \approx F\left(X_{2}, F\left(X_{1}, Y\right)\right)
$$

(the exponential law, $Y^{X_{1} \times X_{2}}=\left(Y^{X_{1}}\right)^{X_{2}}$ ) is a $P V G$ isomorphism for any object $Y$ from $B$ and for any objects $X_{1}$ and $X_{2}$ from $A$ such that $X_{1} \times X_{2}$ is also an object from $A$. We say that $\Psi$ possesses the property (IMB) if, for any $X$ from $A$ and for any $Y_{1}, Y_{2}$ from $B$,

$$
Y_{1} \subset Y_{2} \Rightarrow \Psi\left(X, Y_{1}\right) \subset \Psi\left(X, Y_{2}\right) .
$$

Here, "imbedding" $X_{1} \subset X_{2}$ means that $X_{1}$ is a vector subspace in $X_{2}$ and that the pseudo-topology of $X_{1}$ coincides with the pseudo-topology induced from $X_{2}$.

DEFINITION 2.6. We say that a pseudo-topologizer $\Psi$ on $A \times B$ possesses the property (SAT) if the following condition is fulfilled: for any $X$ from $A$ and any $Y$ from $B$,

$$
\mathfrak{f} \downarrow \Psi(X, Y) \Longleftrightarrow \hat{\mathfrak{f}} \downarrow \Psi(X, Y),
$$

where the "saturation" $\hat{f}$ of a filter $\mathfrak{f}$ in $F(X, Y)$ is defined as the filter generated by the filter basis $\{\hat{F} \mid F \in \mathfrak{f}\}$, the set $\hat{F}$ being for any $F \subset F(X, Y)$ defined by the formula

$$
\hat{F}:=\{f \in F(X, Y) \mid \forall x \in X, f(x) \in F(x)\} .
$$

(The fact that the sets $\hat{F}, F \in \mathfrak{f}$, are really a filter basis follows from the relation $\hat{F}_{1} \cap \hat{F}_{2} \supset$ $\left(F_{1} \cap F_{2}\right)^{\wedge}$.) 
THEOREM 2.7. For any linear filter system $S$, the pseudo-topologizers $F_{S}$ and $F_{S}^{\#}$ possess the properties (EXP), (IMB), and (SAT).

Proof. The assertion on (EXP) and (IMB) was proved in [1, Thm. 1.41]. Let us prove the assertion on (SAT). It is clear from (2.4) that

$$
\hat{F}(U)=F(U) \quad \forall F \subset F(X, Y) \forall U \subset X .
$$

It follows at once from (2.5) that for any filter $f$ in $F(X, Y)$, any filter $x$ in $X$, and any set $U$ in $X$, we have

$$
\begin{aligned}
\hat{\mathfrak{f}}(x) & =\mathfrak{f}(x), \\
\hat{\mathfrak{f}}(U) & =\mathfrak{f}(U) .
\end{aligned}
$$

The fact that $\mathfrak{f} \downarrow F_{S}(X, Y) \Rightarrow \hat{\mathfrak{f}} \downarrow F_{S}(X, Y)$ follows now from (2.6) and Definition 2.3. That $\mathfrak{f} \downarrow F_{S}^{\#}(X, Y) \Rightarrow \hat{\mathfrak{f}} \downarrow F_{S}^{\#}(X, Y)$ follows from (2.7) and the following characterization of convergence in $F_{S}^{\#}(X, Y)$ (see [1, Lem. 1.40]):

$$
\mathfrak{f} \downarrow F_{S}^{\#}(X, Y) \Longleftrightarrow \forall x \in S(X) \exists \mathfrak{q} \downarrow Y^{\#} \forall V \in \mathfrak{y} \exists U \in x \quad f(U) \supset \mathbf{r} V .
$$

This completes the proof.

3. $\Psi$-bounded convexity and $\Psi$-Lipschitz-continuity. Here, we introduce the notions of bounded convexity, $\Psi$-bounded convexity, and $\Psi$-Lipschitz-continuity and recall the notion of $\Psi$-differentiability.

DEFINITION 3.1. Let $\Psi$ be a pseudo-topologizer on a category $A$ of p.v.g.'s containing $\mathbb{R}$ as an object, and let $X$ be a p.v.g. We say that a convex function $f: X \rightarrow \mathbb{R}$ is boundedly convex if there exists a continuous homogeneous function of degree 2 $q: X \rightarrow \mathbb{R}$ such that for any $x_{1}, x_{2}$ from $X$ and any nonnegative numbers $\lambda_{1}, \lambda_{2}$ with $\lambda_{1}+\lambda_{2}=1$,

$$
(0 \leq) \lambda_{1} f\left(x_{1}\right)+\lambda_{2} f\left(x_{2}\right)-f\left(\lambda_{1} x_{1}+\lambda_{2} x_{2}\right) \leq \lambda_{1} \lambda_{2} q\left(x_{2}-x_{1}\right) .
$$

If, in addition, $q$ is a bounded point in $\Psi(X, \mathbb{R})$, we say that $f$ is $\Psi$-boundedly convex.

REMARK 3.2. For any nonnegatively definite quadratic form $(f(x)=b(x, x)$, where $b$ is a symmetric bilinear form, such that $b(x, x) \geq 0$ for all $x)$,

$$
\lambda_{1} f\left(x_{1}\right)+\lambda_{2} f\left(x_{2}\right)-f\left(\lambda_{1} x_{1}+\lambda_{2} x_{2}\right)=\lambda_{1} \lambda_{2} f\left(x_{1}-x_{2}\right)(\geq 0),
$$

so that such forms satisfy condition (3.1) with the last " $\leq$ " changed by "=" and with $q=f$.

REMARK 3.3. It is evident that the addition, to $f$, of a constant or a linear function does not disturb the validity of (3.1), and that a translation of $f$ by any vector $h$ (that is, the pass from $f$ to the function $x \mapsto f(x-h)$ ) also does not disturb (3.1). 
REMARK 3.4. It is easy to see that for normed spaces $X$, the definition of a boundedly convex function reduces to the usual one given in the introduction.

DefinITION 3.5. Let $\Psi$ be a pseudo-topologizer on $A \times B$, and let $X$ and $Y$ be p.v.g.'s from $A$ and $B$, respectively. We say that a mapping $f: X \rightarrow Y$ is $\Psi$-Lipschitz-continuous if the set $f_{\mathbb{R}^{+}, X}$ is bounded in $\Psi(X, Y)$, that is

$$
\mathbf{r} f_{\mathbb{R}^{+}, X} \downarrow \Psi(X, Y),
$$

where

$$
f_{R^{+}, X}=\left\{f_{t, x} \mid t \in \mathbb{R}^{+}, x \in X\right\},
$$

$f_{t, x}$ being (for $t \in \mathbb{R}^{+}$and $x \in X$ ) a mapping from $X$ into $Y$ defined as follows: $f_{t, x}=0$ if $t=0$, and

$$
f_{t, x}(h)=\frac{f(x+t h)-f(x)}{t} \quad \text { if } t>0 .
$$

This definition is indeed an extension of the usual one that is seen from the following lemma.

LEMMA 3.6. Let $X$ and $Y$ be normed spaces. Then a mapping $f: X \rightarrow Y$ is $\Psi_{b}$ Lipschitz-continuous if and only if $f$ is Lipschitz continuous in the usual sense, that is, if and only if there exists $M>0$ such that for any $x_{1}, x_{2}$ from $X$,

$$
\left\|f\left(x_{2}\right)-f\left(x_{1}\right)\right\| \leq M\left\|x_{2}-x_{1}\right\| .
$$

Proof. First of all, notice that equation (3.3) for $\Psi=\Psi_{b}$ means that for every bounded set $B$ in $X$, the set

$$
f_{\mathbb{R}^{+}, X}(B)=\left\{\frac{f(x+t h)-f(x)}{t} \mid t \in \mathbb{R}^{+}, x \in X, h \in B\right\}
$$

is bounded in $\mathrm{Y}$.

Now, let $f$ satisfy (3.6). Then for each $t \in \mathbb{R}^{+}$and each $x \in X$, we have

$$
\left\|\frac{f(x+t h)-f(x)}{t}\right\| \leq \frac{1}{t} M\|t h\|=M\|h\|,
$$

so that, any bounded $B$, the set (3.7) is bounded in $Y$.

Conversely, let the set (3.7) be bounded for any bounded $B$. Take the unit ball as $B$. Let the norms of all elements of the corresponding set (3.7) do not exceed $M$. Then for any $x$ and $h$,

$$
\|f(x+h)-f(x)\| \leq M\|h\| .
$$

Indeed, any $h$ can be written in the form $h=\|h\| e$, where $\|e\|=1$. Without loss of generality, we can assume that $\|h\|=: \alpha>0$. So, we have

$$
\|f(x+h)-f(x)\|=\alpha\left\|\frac{f(x+\alpha e)-f(x)}{\alpha}\right\| \leq \alpha M=M\|h\| .
$$


Definition 3.7 [4]. Let $\Psi$ be a pseudo-topologizer on $A \times B$ and let $X$ and $Y$ be p.v.g.'s from $A$ and $B$, respectively. We say that a mapping $f: X \rightarrow Y$ is $\Psi$-differentiable at a point $x \in X$ if $f$ admits the representation

$$
f(x+h)=f(x)+f^{\prime}(x) h+r(h) \quad(h \in X),
$$

where $f^{\prime}(x) \in L(X, Y)$ (the derivative of $f$ at $\left.x\right)$ and $r$ satisfies the condition

$$
r_{t} \longrightarrow 0 \quad \text { in } \Psi(X, Y) \text { as } t \rightarrow+0,
$$

that is,

$$
r_{\mathbf{r}^{+}} \downarrow \Psi(X, Y),
$$

where $r_{t}$ (for $t \in \mathbb{R}^{+}$) is a mapping from $X$ into $Y$ defined as follows: $r_{t}=0$ if $t=0$, and

$$
r_{1}(h):=\frac{r(t h)}{t} \quad \text { if } t>0
$$

Notice that $F_{B}$-differentiability (respectively, $F_{C}$-differentiability) is the so-called Frölicher-Bucher (respectively, Michal-Bastiani) differentiability and that, for normed spaces, $F_{b}$-differentiability is just Fréchet differentiability.

REMARK 3.8. As shown in [2], for the case of topological vector spaces $F_{C}^{\#}$-differentiability coincides with $F_{B}^{\#}$-differentiability. This is the so-called Hyers-Lang differentiability.

4. The main results. Here is the exact formulation of our results. Comparing with the above roughly speaking formulation in the introduction, a condition of continuity appears now twice.

THEOREM 4.1. Let $\Psi$ be a pseudo-topologizer on $P L G \times P L G$ that possesses the properties (EXP), (IMB), and (SAT). Let $X$ be an arbitrary p.v.s. If a continuous convex function $f: X \rightarrow \mathbb{R}$ is $\Psi$-boundedly convex, the corresponding function $q$ (see Definition 3.1) being also continuous, then $f$ is everywhere $\Psi$-differentiable and its derivative $f^{\prime}: X \rightarrow$ $L(X, \mathbb{R})$ is $\Psi$-Lipschitz continuous, $L(X, \mathbb{R})$ being supplied with the pseudo-topology induced from $\Psi(X, \mathbb{R})$.

Proof. We have the following steps.

STEP 1. Here we show that $f$ is everywhere Gateaux differentiable. Since $f$ is convex, the restriction of $f$ onto each straight line is a convex continuous function. As is wellknown (see, e.g., [7]), for this restriction, there exist both one-sided derivatives at each point of the straight line. This means that our function $f$ is differentiable at each point $x$ in any direction $h$. Denote the corresponding mapping

$$
h \longmapsto d_{h} f(x):=\lim _{t \downarrow 0} \frac{f(x+t h)-f(x)}{t}, \quad X \rightarrow Y
$$

by $f^{\prime}(x)$. We need to verify that this mapping is linear and continuous. It is evident that it is positively homogeneous. By Remark 3.3, we may assume that $x=0$ and $f(0)=0$. 
STEP 2. Now, we show that the mapping $f^{\prime}(0)$ is linear. Put $x_{1}=t h_{1}, x_{2}=t h_{2}(0<$ $\left.t<1, h_{1}, h_{2} \in X\right)$ in (3.1) and divide by $t$ :

$$
\begin{aligned}
0 & \leq \lambda_{1} \frac{f\left(t h_{1}\right)}{t}+\lambda_{2} \frac{f\left(t h_{2}\right)}{t}-\frac{f\left(t\left(\lambda_{1} h_{1}+\lambda_{2} h_{2}\right)\right)}{t} \\
& \leq \frac{1}{t} \lambda_{1} \lambda_{2} q\left(t\left(h_{2}-h_{1}\right)\right)=t \lambda_{1} \lambda_{2} q\left(h_{2}-h_{1}\right),
\end{aligned}
$$

(we have used the fact that $q$ is homogeneous of degree 2). As $t \downarrow 0$, we obtain

$$
0 \leq \lambda_{1} f^{\prime}(0) h_{1}+\lambda_{2} f^{\prime}(0) h_{2}-f^{\prime}(0)\left(\lambda_{1} h_{1}+\lambda_{2} h_{2}\right) \leq 0 \text {. }
$$

So,

$$
f^{\prime}(0)\left(\lambda_{1} h_{1}+\lambda_{2} h_{2}\right)=\lambda_{1} f^{\prime}(0) h_{1}+\lambda_{2} f^{\prime}(0) h_{2}
$$

for all $h_{1}, h_{2}$ from $X$ and all nonnegative $\lambda_{1}, \lambda_{2}$ with $\lambda_{1}+\lambda_{2}=1$. If we take $h_{1}=-h_{2}=$ $-h \cdot \lambda_{1}=\lambda_{2}=1 / 2$, we obtain

$$
f^{\prime}(0)(-h)=-f^{\prime}(0) h
$$

$\left(f^{\prime}(0) 0=0\right.$ by the mentioned homogeneity of $\left.f^{\prime}(0)\right)$. The desired linearity follows from (4.4), (4.5), and from the homogeneity of $f^{\prime}(0)$.

STEP 3. Here, we verify that $f^{\prime}(0)$ is continuous. Take $x_{1}=0, x_{2}=h, \lambda_{1}=1-t, \lambda_{2}=$ $t(0<t<1, h \in X)$ in (3.1):

$$
0 \leq t f(h)-f(t h) \leq t(1-t) q(h),
$$

whence it follows that

$$
0 \leq-\frac{f(t h)}{t}+f(h) \leq(1-t) q(h)
$$

As $t \downarrow 0$, we obtain

$$
0 \leq-f^{\prime}(0) h+f(h) \leq q(h) .
$$

If $h \rightarrow 0$, then $f(h)$ and $q(h)$ tend to zero by the supposed continuity of $f$ and $q$. Hence, $f^{\prime}(0) h \rightarrow 0$ if $h \rightarrow 0$, that is, $f^{\prime}(0)$ is continuous at 0 and, thereby, everywhere.

Thus, we have proved that $f$ is everywhere Gateaux differentiable.

STEP 4. Now, we prove that $f$ is $\Psi$-differentiable at each point $x$. By Remark 3.3 and the fact that the addition of continuous affine functions and translations do not disturb $\Psi$-differentiability, we may assume that $x=0, f(0)=0, f^{\prime}(0)=0$ (where $f^{\prime}(0)$ is the Gateaux derivative at 0 which was proved to exist). We need to verify that

$$
r_{t} \longrightarrow 0 \quad \text { in } \Psi(X, Y) \text { as } t \downarrow 0,
$$

where

$$
r_{t}: h \longmapsto \frac{f(t h)-f(0)}{t}=\frac{f(t h)}{t}, \quad X \rightarrow Y .
$$

Take $x_{1}=-t h, x_{2}=t h,(0<t<1, h \in X)$ in (3.1)

$$
0 \leq \frac{1}{2} f(-t h)+\frac{1}{2} f(t h) \leq \frac{1}{4} q(2 t h)=t^{2} q(h) .
$$


Since the function $f$ is convex and $f(0)=0$ and $f^{\prime}(0)=0$, both the values $f(-t h)$ and $f(t h)$ are nonnegative. Hence,

$$
0 \leq \frac{f(t h)}{t} \leq 2 t q(h)
$$

that is,

$$
0 \leq r_{t} \leq 2 t q
$$

If $t \downarrow 0$, then $t q \rightarrow 0$ in $\Psi(X, Y)$ since $q$ is $\Psi$-bounded. Therefore, also $r_{t} \rightarrow 0$ in $\Psi(X, Y)$.

STEP 5. Now, we show that, for any $x_{1}, x_{2}$ from $X$,

$$
0 \leq f\left(x_{2}\right)-f\left(x_{1}\right)-f^{\prime}\left(x_{1}\right) \cdot\left(x_{2}-x_{1}\right) \leq q\left(x_{2}-x_{1}\right) .
$$

Again, we may assume, without loss of generality, that $x=0, f(0)=0$, and $f^{\prime}(0)=0$ (since equation (4.8) does not disturb by the addition to $f$ of constants and linear functions). The relation to be proved takes then the form (if we put $x_{2}=h$ )

$$
0 \leq f(h) \leq q(h)
$$

for any $h \in X$. But this follows at once from (4.8).

STEP 6. Now, we go to the proof of the main assertion on $\Psi$-Lipschitz continuity of our derivative. We need to show that

$$
\mathbf{r} f_{\mathbb{R}^{+}, X}^{\prime} \downarrow \Psi\left(X, L(X, \mathbb{R})_{\Psi}\right) .
$$

By the properties (EXP) and (IMB) which are fulfilled for $\Psi$ by the assumption, equation (4.16) is equivalent to the relation

$$
\mathbf{r} \overline{f_{\mathbb{R}^{+}, X}^{\prime}} \downarrow \Psi(X \times X, \mathbb{R}),
$$

where $\overline{f_{\mathbb{R}^{+}, X}^{\prime}}$ is the set in $F(X \times X, \mathbb{R})$, that corresponds to the set $f_{\mathbb{R}^{+}, X}^{\prime}$ by the canonical isomorphism

$$
F(X, F(X, \mathbb{R}))=F(X \times X, \mathbb{R}) .
$$

In the next step, we show that

$$
\overline{f_{\mathbb{R}^{+}, X}^{\prime}} \subset I_{1}\left(\frac{1}{2} q \circ \pi_{1}+2 q \circ \pi_{2}\right),
$$

where $I_{1}=[-1,1]$, and $\pi_{1}$ and $\pi_{2}$ are the canonical projections of the product $X \times X$ onto the factors. It follows from (4.19) that

$$
\mathbf{r} \overline{f_{\mathbb{R}^{+}, X}^{\prime}} \supset \mathbf{r}\left(\frac{1}{2} q \circ \pi_{1}+2 q \circ \pi_{2}\right) \supset(\mathbf{r} q) \circ \pi_{1}+(\mathbf{r} q) \circ \pi_{2} .
$$

But $\mathbf{r} q \downarrow \Psi(X, \mathbb{R})$ by the fact that $q$ is $\Psi$-bounded. Hence, both the terms in the righthand side of (4.20) converge to 0 in $F(X \times X, \mathbb{R})$ by condition (b) of Definition 2.1. Thereby, (4.17) is proved.

STEP 7. It remains to show equation (4.19). We have, for $t \in \mathbb{R}^{+}$and $x, h_{1}, h_{2} \in X$,

$$
\overline{f_{t, x}^{\prime}}\left(h_{1}, h_{2}\right)=f_{t, x}^{\prime}\left(h_{1}\right) \cdot h_{2}=\frac{f^{\prime}\left(x+t h_{1}\right)-f^{\prime}(x)}{t} \cdot h_{2} \text {. }
$$

(Here, the bar is to be understood in the same sense as in equation (4.17) above.) Put 
$x_{1}=x, x_{2}=x+t h_{1}, x_{3}=x+t h_{2}, x_{4}=x+t h_{1}-t h_{2}, x_{0}=x+(1 / 2) t h_{1}$, so that $x_{0}$ is the center of the parallelogram with the vertices $x_{1}, \ldots, x_{4}$. By (3.1),

$$
\frac{1}{2} f\left(x_{1}\right)+\frac{1}{2} f\left(x_{2}\right)-f\left(x_{0}\right) \leq \frac{1}{4} q\left(t h_{1}\right)=\frac{1}{4} t^{2} q\left(h_{1}\right) .
$$

By convexity of $f$,

$$
0 \leq \frac{1}{2} f\left(x_{3}\right)+\frac{1}{2} f\left(x_{4}\right)-f\left(x_{0}\right) .
$$

By (4.14),

$$
\begin{aligned}
& f\left(x_{3}\right)-f\left(x_{1}\right)-f^{\prime}\left(x_{1}\right) \cdot\left(+t h_{2}\right) \leq q\left(t h_{2}\right)=t^{2} q\left(h_{2}\right), \\
& f\left(x_{4}\right)-f\left(x_{2}\right)-f^{\prime}\left(x_{2}\right) \cdot\left(-t h_{2}\right) \leq q\left(t h_{2}\right)=t^{2} q\left(h_{2}\right) .
\end{aligned}
$$

If we take the sum of the four inequalities from (4.22) to (4.25), the first two being multiplied by 2 and -2 , respectively, then we obtain

$$
f^{\prime}\left(x_{2}\right) \cdot t h_{2}-f^{\prime}\left(x_{1}\right) \cdot t h_{2} \leq \frac{t^{2}}{2} q\left(h_{1}\right)+2 t^{2} q\left(h_{2}\right),
$$

whence it follows that

$$
\frac{1}{t}\left[f^{\prime}\left(x+t h_{1}\right)-f^{\prime}(x)\right] \cdot h_{2} \leq \frac{1}{2} q\left(h_{1}\right)+2 q\left(h_{2}\right) .
$$

If we substitute here $h_{2}$ by $-h_{2}$, then we find that the left-hand side of (4.27) times -1 also does not exceed the right-hand side of (4.27). So, the left-hand side belongs to $I_{1}\left(1 / 2 q\left(h_{1}\right)+2 q\left(h_{2}\right)\right)$. Therefore, (see (4.21))

$$
\overline{f_{t, x}^{\prime}}\left(h_{1}, h_{2}\right) \in I_{1}\left(\frac{1}{2} q\left(h_{1}\right)+2 q\left(h_{2}\right)\right)=I_{1}\left(\frac{1}{2} q \circ \pi_{1}+2 q \circ \pi_{2}\right)\left(h_{1}, h_{2}\right) .
$$

So, if we put, for short,

$$
\frac{1}{2} q \circ \pi_{1}+2 q \circ \pi_{2}=: p
$$

then

$$
\forall h_{1}, h_{2} \quad \overline{f_{t, x}^{\prime}}\left(h_{1}, h_{2}\right) \in I_{1} p\left(h_{1}, h_{2}\right),
$$

whence it follows that

$$
\forall t, \alpha \in \mathbb{R}^{+} \forall x, h_{1}, h_{2} \in X, \quad I_{\alpha} \overline{f_{t, x}^{\prime}}\left(h_{1}, h_{2}\right) \subset I_{\alpha} p\left(h_{1}, h_{2}\right) .
$$

Therefore,

$$
\mathbb{R} \overline{f_{\mathbb{R}^{+}, X}^{\prime}} \supset(\mathbf{r} p)^{\wedge} .
$$

But $(\mathbf{r} p)^{\wedge} \downarrow \Psi(X, \mathbb{R})$ since $\mathbf{r} p \downarrow \Psi(X, \mathbf{r})$, and $\Psi$ satisfies (SAT). Hence, $\mathbf{r} \overline{f_{\mathbb{R}^{+}, x}^{\prime}} \downarrow \Psi(X, \mathbb{R})$. The theorem is proved.

COROLLARY 4.2. Let $S$ be a linear filter system in a category $A$ of p.v.g.'s, let $X$ be a p.v.g. from $A$, and let $f: X \rightarrow \mathbb{R}$ be a continuous convex function. If $f$ is $F_{s}$-boundedly convex, then $f$ is everywhere $F_{s}^{\#}$-differentiable and its derivative $f^{\prime}: X \rightarrow L(X, \mathbb{R})$ is $F_{S}$-Lipschitz-continuous, $L(X, \mathbb{R})$ being supplied with the pseudo-topology induced from $F_{s}^{\#}(X, \mathbb{R})$. 
Proof. If the function $q$ that appears in Definition 3.1 is a bounded point in $F_{S}(X, \mathbb{R})$, then $q$ is also a bounded point in $F_{S}^{\#}(X, \mathbb{R})$ since $\mathbf{r} q \downarrow F_{S}(X, \mathbb{R}) \Rightarrow \mathbf{r} q \downarrow F_{S}^{\#}(X, \mathbb{R})$ by the definition of the onion modification. So, the assertion of Corollary 4.2 follows from Theorem 4.1.

COROLlary 4.3. Let $X$ be a p.v.g., and let $f: X \rightarrow \mathbb{R}$ be a continuous convex function. If $f$ is boundedly convex, then $f$ is everywhere Hyers-Lang differentiable and its derivative $f^{\prime}: X \rightarrow L(X, \mathbb{R})$ is $F_{C}^{\#}$-Lipschitz-continuous, $L(X, \mathbb{R})$ being supplied with the pseudo-topology induced from $F_{C}^{\#}(X, \mathbb{R})$.

Proof. This follows from Lemma 2.4 and Remark 3.8.

REMARK 4.4. Since $F_{C}^{\#}$ coincides, for normed spaces, with $f_{b}$ (see [2]) and HyersLang differentiability coincides, for normed spaces, with Fréchet differentiability (see [3]), Corollary 4.3 reduces, in the case of a normed space $X$, (in view of Lemma 3.6) to the result of Focke [5].

\section{REFERENCES}

[1] V. I. Averbuch, The continuity of a composition, Math. Nachr. 75 (1976), 153-183 (Russian). MR 55 6469. Zbl 364.46054.

[2] _ _ Pseudotopologizers and polylinear mappings, Math. Nachr. 114 (1983), 343-368 (Russian). MR 86m:46041. Zbl 592.46061.

[3] V. I. Averbuch and O. G. Smoljanov, Different definitions of derivative in linear topological spaces, Uspekhi Mat. Nauk 23 (1968), no. 4, 67-116 (Russian). MR 39\#7424. Zbl 179.19103.

[4] _ Pseudo-topologies and differentiation, Vestnik Moskov. Univ. Ser. I Mat. Mekh. 27 (1972), no. 2, 3-9 (Russian). MR 45 9131. Zbl 244.46057.

[5] J. Focke, Beschränkt konvexe Funktionen und die Lipschitz-Stetigkeit ihres Gradienten, Math. Operationsforsch. Statist. Ser. Optim. 8 (1977), no. 4, 505-507. MR 5717430. Zbl 386.46038.

[6] A. Frölicher and W. Bucher, Calculus in Vector Spaces without Norm, Lecture Notes in Mathematics, no. 30, Springer-Verlag, Berlin, New York, 1966. MR 35\#4723. Zbl 156.38303.

[7] R. T. Rockafellar, Convex Analysis, Princeton Mathematical Series, no. 28, Princeton University Press, Princeton, NJ, 1970. MR 43\#445. Zbl 193.18401.

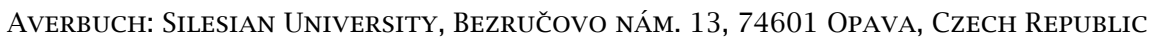

E-mail address: V1adimi r. Averbuch@fpf.slu.cz 


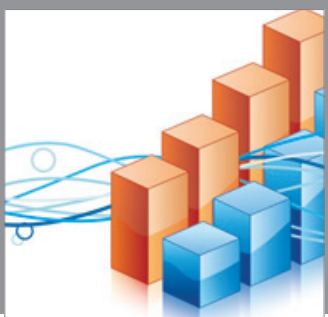

Advances in

Operations Research

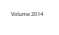

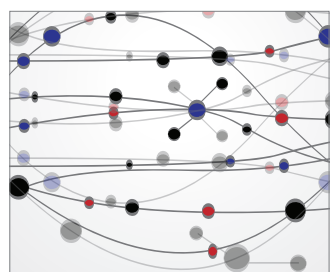

\section{The Scientific} World Journal
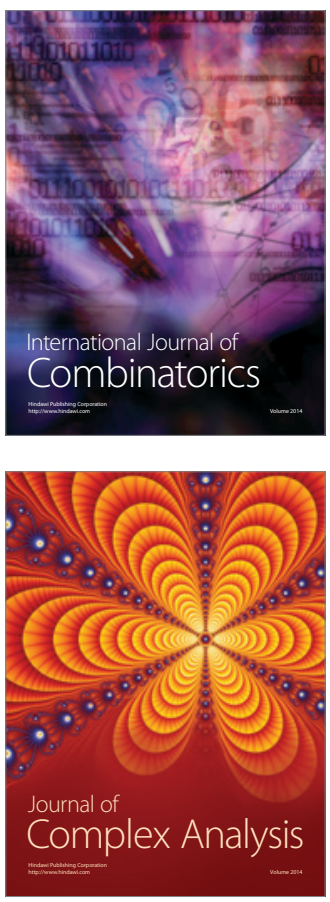

International Journal of

Mathematics and

Mathematical

Sciences
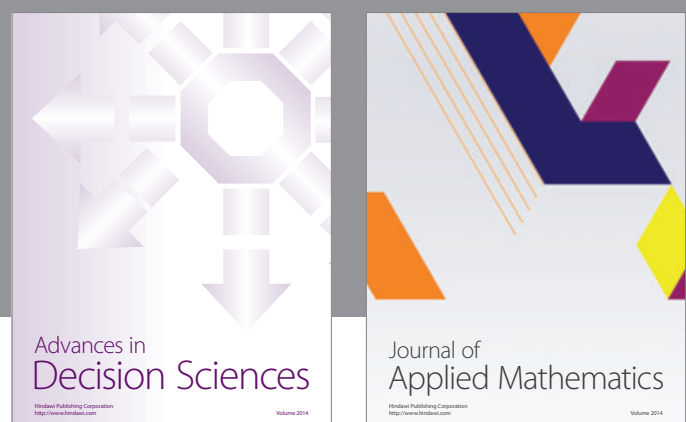

Journal of

Applied Mathematics
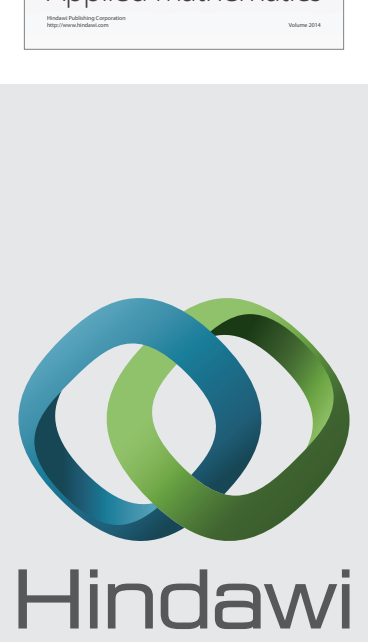

Submit your manuscripts at http://www.hindawi.com
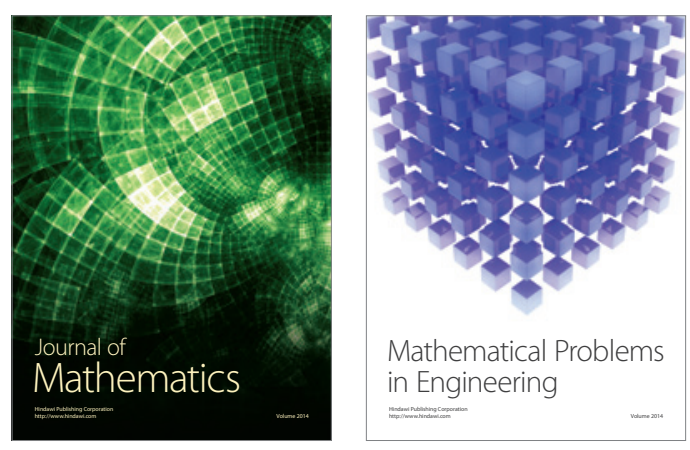

Mathematical Problems in Engineering
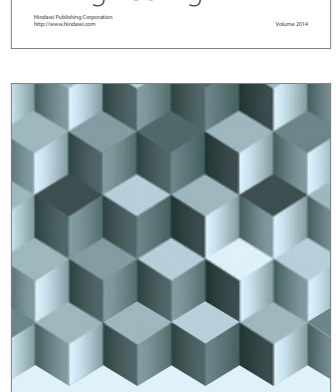

Journal of

Function Spaces
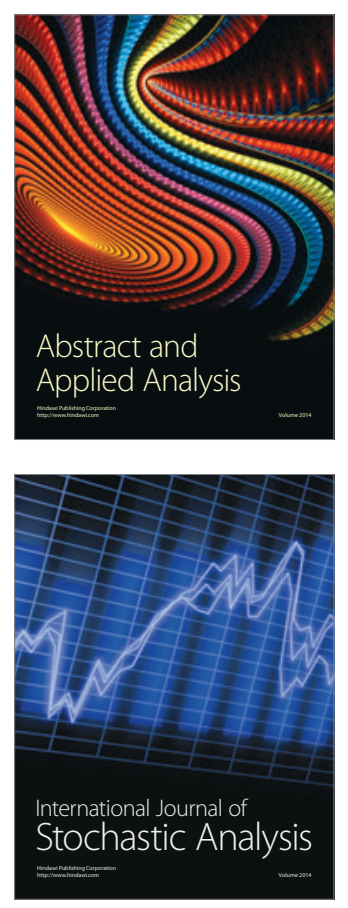

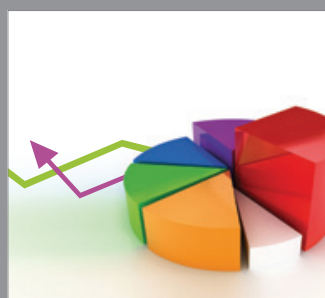

ournal of

Probability and Statistics

Promensencen
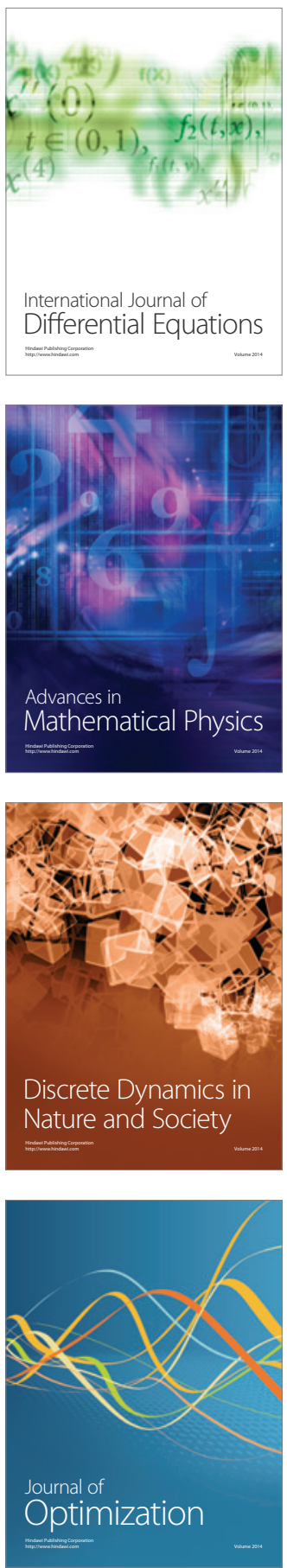